\title{
ACERCA DE LAS METODOLOGIAS DE INTERVENCIÓN EN TRABAJO SOCIAL
}




\title{
RESUMEN
}

\begin{abstract}
El artículo busca aportar al debate acerca de las metodologías de intervención en Trabajo Social partiendo de la hipótesis de que la crisis societal actual, demanda nuevas formas de acción profesional, teniendo en consideración los procesos de Trabajo Social en los distintos campos de desempeño profesional en la sociedad contemporánea, lo cual requiere un análisis riguroso del objeto de Trabajo Social , las formas como ha desarrollado su contenido teórico-metodológico y de la finalidad de la intervención profesional.
\end{abstract}

\section{INTRODUCCIÓN}

1 presente trabajo tiene como objetivo contribuir a la discusión sobre la práctica profesional del Trabajo Social. Interesa, específicamente, aportar al debate acerca de las metodologías de intervención en Trabajo Social.

En principio, entiendo que existe consenso entre los-las profesionales de Trabajo Social, acerca de la existencia de tres clásicas metodologías de intervención en Trabajo Social $^{1}$ : caso (individuo-familia), grupo y comunidad, y que cada una de ellas posee un conjunto de técnicas (instrumentos y herramientas) útiles para la aproximación a dichas realidades.

Sin embargo, dada la posibilidad y la necesidad de abrir espacios de discusión, lo que se intentará sustentar es este documento es que la crisis societal actual, demanda nuevas formas de acción profesional, teniendo en consideración los procesos de trabajo del Trabajo Social en los distintos campos de desempeño profesional, en la sociedad contemporánea, lo cual requiere un análisis riguroso del objeto del Trabajo Social, las formas como ésta ha desarrollado su "arsenal" teórico-metodológico y de la finalidad de la intervención profesional. Dicho análisis, principalmente, deberá estar soportado en la investigación.

El documento se ha dividido en tres partes. En la primera parte, se presentan algunas aproximaciones al objeto de intervención propio del Trabajo Social, para sustentar porqué los cambios actuales demandan redefiniciones y exigen la de-construcción teórico-

${ }^{1}$ Algunos estudiosos del tema reclaman que dichas metodologías, realmente, son métodos, porque éstos dan cuenta de "procedimientos empleados para alcanzar un fin". 
metodológica. Posteriormente, se discute someramente qué entendemos por metodología de intervención.

Después, se abordan los cambios societarios actuales y su repercusión sobre el Trabajo Social: formación, proceso de trabajo y mercado laboral, tomando como ejemplo el área de la salud.

Por último, se esbozan algunas sugerencias para fortalecer el abordaje del asunto de las metodologías de intervención profesional en la formación profesional.

Se resalta, que este documento fue escrito en el marco de la convocatoria docente de la Escuela de Trabajo Social y Desarrollo Humano de la Universidad del Valle - Cali, y es fruto de reflexiones que la autora ha venido realizando, en tanto se desempeña como investigadora y profesional de Trabajo Social en el campo de la salud.

\section{LAS PARTICULARIDADES DEL TRABAJO SOCIAL:}

La aproximación conceptual a cualquier tema, se hace con base en un referente teórico y desde una postura ideológica. En este caso, antes de aproximarnos al tema de las metodologías de intervención en Trabajo Social, consideramos necesario "definir" qué entendemos por Trabajo Social e identificar cuál es su objeto de intervención y, a partir de ahí, adentrarnos en el asunto de las metodologías.

Corresponde, entonces, precisar los dos ámbitos desde los cuales se debe entender el Trabajo Social:

1. Como una profesión que se inscribe en la división social y técnica del trabajo colectivo; es decir, es un tipo de trabajo que responde a unas necesidades sociales, traducidas como demandas a la profesión. Lo cual significa que la profesión no se transforma, ni se madura autónomamente. Por el contrario, las transformaciones societales provocan cambios en la profesión, los cuales se manifiestan en las modificaciones en su objeto de intervención, en nuevas demandas de atención y en alteración en las condiciones de trabajo.

2. El espacio socio-ocupacional o campo de desempeño profesional del Trabajo Social, se circunscribe a la llamada "cuestión social", y una de las formas de enfrentar la "cuestión social" es a través de las políticas sociales.

En el primer enunciado, al inscribir el Trabajo Social en la división socio-técnica del trabajo, se ubica a éste como una profesión vinculada al conjunto de relaciones y condiciones sociales y se rompe con la tendencia de enfocar el Trabajo Social en si mismo (visión endógena de la profesión)², al relacionarlo con procesos sociales más amplios. Además, posibilita abordar su especificidad frente a otras profesiones que hacen, también, parte de la división socio-técnica del trabajo colectivo.

2 Iamamoto, Marilda V. O Serviço Social na Contemporaneidade: trabalbo e formaçao profissional. Cortez Editora. São Paulo, 2001 
Adicionalmente, dicha perspectiva permite entender que la población coloca demandas o mejor sus necesidades se traducen en demandas a las profesiones. Es decir, la población participa de la configuración de los campos de trabajo de las distintas profesiones. Por tanto, significa que el Trabajo Social, como profesión, se crea sustentado en las necesidades sociales y se desarrolla a partir de la legitimidad que le da socialmente su utilidad.

“...el fundamento de la existencia de una profesión se localiza en las demandas histórico-sociales y en la capacidad de responder a ellas” (Guerra, Y. Ibíd.)

En el segundo enunciado, se parte de entender la "cuestión social" como la expresión politizada de las desigualdades sociales. Ello sitúa al Trabajo Social como una profesión directamente vinculada con la realidad: "el Trabajo Social no actúa solamente sobre la realidad, sino en la realidad".

A partir de las dos consideraciones, nos interesa destacar que las profesiones, en este caso el Trabajo Social, no puedan ser entendidas ajenas a los cambios macro-societarios, pues éstos repercuten directa e indirectamente sobre ellas y, por lo tanto, el asunto de las metodologías en la actualidad sólo podrá abordarse críticamente cuando se articule al análisis de los procesos sociales contemporáneos.

Además, supone que es la investigación de la realidad lo que permite la construcción de nuevos caminos para la práctica profesional y para la formulación de propuestas que sobrepasen el discurso genérico, que no da cuenta de las situaciones particulares, con las cuales lidian cotidianamente los y las trabajadores sociales ${ }^{3}$.

Ambos enunciados, nos llevan a concluir que frente a un momento de cambio o de crisis como el actual ${ }^{4}$, se hace necesario aprehender sus especificidades (sociales, políticas, económicas y culturales) y el reto está en identificar y comprender cómo se traduce en la particularidad práctico-social del Trabajo Social, el impacto de las transformaciones societarias; es decir, cuáles son las mediaciones que conectan la profesión a dichas transformaciones ${ }^{5}$.

3 Iamamoto, Marilda V. O Serviço Social na Contemporaneidade: trabalho e formaçao profissional. Cortez Editora. São Paulo, 2001.

${ }^{4}$ Nos referimos especialmente a los procesos de reestructuración productiva, globalización y hegemonía neoliberal, momentos constitutivos de la coyuntura actual.

${ }^{5}$ Netto, J.P. Transformacões societarias e serviço social: notas para uma análise prospectiva da profissão no Brasil. Revista Serviço Social \& Sociedade. Ano XVII, Abril 1996. Cortez Editora, São Paulo. O Serviço Social no Século XXI. 


\section{POR QUÉ PENSAR EN LAS METODOLOGÍAS DE INTERVENCIÓN EN TRABAJO SOCIAL:}

La preocupación por el asunto de las metodologías de intervención en Trabajo Social no es reciente. Aún, después del proceso de reconceptualización, no se ha logrado superar la "angustia" por la falta o por la necesidad de metodologías propias, las cuales le garantizarían el "status" de profesión".

Quizás al concebir al Trabajo Social como una técnica social, por su carácter de profesión fundamentalmente interventiva, el asunto de las metodologías y los modelos de intervención gana relevancia. Sin embargo, ello supone localizar la "cientificidad" de la profesión en su sustento metodológico; es decir, la "cientificidad" del Trabajo Social estaría derivada de la utilización de procedimientos metodológicos correctos y de un sustento teórico que le acompañe.

Cabe resaltar, que entendemos metodología de intervención, no como un conjunto de procedimientos o como medio de aplicación inmediata del conocimiento, sino como una dirección analítica de una práctica. Es decir, una metodología de intervención posee un sustento teórico y su forma más concreta es el método (o los métodos), el cual, a su vez, se materializa en unos momentos o fases y se desarrolla con instrumentos y técnicas.

Por lo tanto, debe entenderse que "entre el conocimiento y la acción hay mediaciones de diferentes naturaleza, sobretodo, determinaciones objetivas de la realidad y subjetivas de los sujetos", y que la teoría no se confunde con una metodología, pues "ella (la teoría) ilumina las estructuras de los procesos sociales, las determinaciones contradictorias de los procesos que constituyen los fenómenos, disuelve la objetividad de los hechos por su negación, pero no ofrece, no se propone los medios o instrumentos profesionales de acción inmediata sobre los fenómenos"8.

Es decir, la metodología no puede ser confundida con el "cómo instrumental", ni es un producto inmediato de la teoría. La metodología, es un proceso que involucra el conocimiento del contexto en el que se realiza la práctica profesional e incorpora las funciones profesionales asignadas institucional o socialmente y los objetivos de la profesión'. Vale destacar, que no es sólo la voluntad individual la que direcciona el quehacer profesional, sino también el proyecto ético-político que el gremio profesional impulsa.

\footnotetext{
${ }^{6}$ Aunque como lo indica Carlos Montaño, el análisis del asunto de las metodologías en trabajo social es necesario y aún inacabado, por lo tanto sigue siendo vigente.

${ }^{7}$ Guerra, Yolanda. Instrumentalidade do processo de trabalho e serviço social. Revista Serviço Social \& Sociedade, Ano XXI, março 2000, No. 62. Processo de Trabalho e asistencia social

${ }^{8}$ Guerra, Yolanda. Ibidem.

${ }^{9}$ Vélez Restrepo, Olga Lucía. Reconfigurando el Trabajo Social: perspectivas y tendencias contemporáneas. Editorial Espacio. Buenos Aires, 2003.
} 
En Colombia, el Trabajo Social actual, en su búsqueda de "cientificidad" para renovar la legitimidad de la práctica profesional, pareciera estar atravesando por un eclecticismo ${ }^{10}$, sobre el cual se sustenta, incorrectamente, que ante la complejidad del mundo actual, se requiere per se una renovación teórico-metodológica.

Lo anterior, supone una visión endógenista de la profesión, ya que dado el origen y el desarrollo del Trabajo Social como profesión, el momento actual requiere, más que nada, el reconocimiento de las condiciones objetivas en las cuales la intervención profesional se realiza. Dichas condiciones objetivas no pueden ser vistas como "paño de fondo" sobre el cual se desarrolla la profesión.

Por el contrario, como fue colocado antes, son dichas condiciones, aunadas a la dinámica propia de la profesión ${ }^{11}$, las que determinan la práctica profesional y, en ella, el "arsenal" teórico-metodológico requerido.

"...las condiciones objetivas colocadas a la intervención profesional no dependen solo de la postura teleológica individual de los agentes, ni de sus instrumentos de intervención..."12.

El actual periodo de transición ${ }^{13}$ en que "lo viejo no murió y lo nuevo aún no nació", caracterizado por ser una etapa de profundas transformaciones no sólo económicopolíticas, sino también culturales, presenta como consecuencias fundamentales la redefinición de las relaciones entre el Estado y la sociedad civil, cambios en el mundo del trabajo y modificaciones en las formas como es enfrentada la cuestión social.

Dichas transformaciones provocan alteraciones en el ejercicio profesional, en los espacios de intervención, modifican las expresiones de la cuestión social (materia prima de la intervención del trabajado social), y atribuyen nuevas funciones a la profesión ${ }^{14}$. Además, alteran las propias condiciones de trabajo de los-las trabajadoras sociales.

\footnotetext{
${ }^{10}$ Veáse, por ejemplo, que en la mayoría de las últimas publicaciones de trabajo social la búsqueda teórica de la profesión pasa por la teoría de la complejidad, la teoría de la comunicación humana, la acción social, la teoría sistémica, la cibercultura, el modelo de las revoluciones científicas, el interaccionismo simbólico, la sociedad del conocimiento, entre otras. Esta "Torre de Babel", a mi modo de ver, se muestra distante de los fenómenos macroscópicos actuales.

${ }^{11}$ Nos referimos a la dinámica que el gremio profesional es capaz o no de imprimirle al curso de la profesión, de acuerdo al proyecto ético-político que se quiera privilegiar. No hablamos de las posturas individuales.

${ }^{12}$ Guerra, Yolanda. A Instrumentalidade do Serviço Social. Cortez Editora. São Paulo, 1995. Pág. 28

${ }^{13}$ Existe una extensa bibliografía sobre el tema de la crisis de final de siglo pasado, entre ellas destacamos la producción de Harvey, D. (1994), Antunes, R. (1995), Ahumada, C.(1996 ) y Stiglitz (2002).

${ }^{14}$ Guerra, Yolanda. A crise contemporânea e os impactos no serviço social. Manuscrito inédito. 2001.
} 
En ese marco, proponemos, pues, adentrarnos a uno de los campos de intervención del Trabajo Social: la producción de servicios de salud, considerándolo como ejemplo "típico", capaz de mostrar cómo las transformaciones actuales vienen modificando la práctica profesional de Trabajo Social y trataremos de elucidar el asunto de las metodologías de intervención en dicho campo.

No es posible hablar en la actualidad de la producción de servicios de salud o del quehacer profesional de Trabajo Social en salud, sin abordar cómo las transformaciones societales que iniciaron al final del siglo pasado, han impactado este campo. Nos referimos, específicamente, al proceso de reforma a los sistemas de salud acontecidos durante los años 90, en casi todo el mundo.

Dicha reforma está relacionada con los cambios en la concepción de salud, en la forma de prestación y administración de los servicios y en el papel de los usuarios en la gestión de los servicios de salud.

La reforma sectorial ha impactado el ejercicio profesional de todas las profesiones vinculadas directa e indirectamente con la producción de servicios de salud y, aunque insuficientemente abordado, el Trabajo Social ha venido siendo reconfigurado ${ }^{15}$.

En Colombia, la reforma ocurrida a partir de la implementación del nuevo sistema de seguridad social en salud -SGSSS-, con la Ley 100 de 1993, se sustentó en la necesidad de la ampliación de la cobertura, la libertad de elección, la productividad, la rentabilidad, la prestación de servicios de acuerdo al régimen de afiliación y el énfasis en la atención a la enfermedad.

Para Trabajo Social, además de que el crecimiento y la intensificación de la pobreza, la emergencia y re-emergencia de enfermedades, la población desplazada, el desempleo, entre otros, le han modificado su objeto de intervención, la reforma sectorial ha significado, también, cambios en el objeto de intervención, en el proceso y en las condiciones de trabajo.

Siendo que se le ha demandado al trabajador-a social entrar en la lógica de la productividad y la eficiencia (indicador de ello es la facturación de cualquier actividad realizada), se vislumbra una burocratización de la práctica profesional, entendida no como una opción del o la profesional, sino como una condición objetiva real.

A mi modo de ver, cuestiones como la pobreza, el derecho a la salud, la gestión de los servicios de salud, la nueva configuración de las políticas sociales, están exigiendo otro "arsenal" teórico-metodológico al trabajador social, que impida que la burocratización, vía la implementación de los llamados protocolos de atención, se imponga sobre las dinámicas sociales particulares.

Ese "arsenal" debe contener un mejor conocimiento de la persona humana (del sujeto social, del usuario) y un mejor análisis de la situación en que se éste se encuentra, así como de las brechas que el actual SGSSS permite. Ello posibilitaría, identificar los límites

\footnotetext{
${ }^{15}$ Existen estudios publicados sobre el tema en Canadá, Estados Unidos y Brasil, principalmente.
} 
y las posibilidades de la actuación profesional, así como evitar posturas acríticas o tecnicistas ${ }^{16}$.

Considero que al recuperar el proceso de trabajo del trabajador-a social en la producción de servicios de salud, en el marco de las actuales transformaciones políticas, sociales, económicas y culturales, se identificarían los determinantes de la práctica profesional y se podrían realizar nuevas propuestas de intervención. De esa forma, se resalta que las metodologías se construyen en función de las particularidades de los temas o campos que son objeto de estudio y acción de los trabajadores sociales.

“... tanto para los procesos de investigación como para los de intervención, es el objeto el que determina el método y no a la inversa"17.

En ese sentido, la investigación de situaciones concretas que son objeto de trabajo de los y las trabajadores-as sociales, es el camino necesario para la comprensión de los fenómenos sociales particulares, con los cuales el-la Trabajadora Social lidia en su cotidiano, alimentando la elaboración de propuestas amarradas a la realidad y capaces de accionar las posibilidades del cambio existentes.

\section{PROPUESTAS}

En términos prácticos y con ánimo propositivo se presentan a continuación sugerencias para alimentar y avanzar, en el ámbito de la formación profesional, en la discusión sobre las metodologías de intervención en Trabajo Social:

1. Propender por el desarrollo de un proyecto de formación profesional, en el que se definan lineamientos curriculares mínimos para Trabajo Social, sobre la base de los desafíos sociales actuales y el compromiso ético-político de la profesión.

Ello supone la revisión de la estructura curricular, de los modelos pedagógicos usados y de los fines de la profesión.

2. De forma preliminar, se sugiere que en el currículo se incluya el análisis de las particularidades del Trabajo Social, a partir de las características que definen e institucionalizan la profesión en los distintos campos de actuación profesional, considerando el marco político, social, económico y cultural actual.

Es decir, se requiere profundizar en el proceso de trabajo del trabajo social, considerando las demandas emergentes en los distintos espacios institucionales (públicos

\footnotetext{
${ }^{16}$ Es común encontrar trabajadoras sociales de los servicios de salud que consideran que su labor es cumplir estrictamente un protocolo de atención y su trato al usuario se hace desde el poder que le da la opción de decidir si se disminuye el costo por un servicio o no. De forma complementaria, está la queja constante por la imposibilidad de hacer "más" ya que el sistema de salud no lo permite.

${ }^{17}$ Aquín, Nora. Por qué desarrollar la especificidad. Revista Prospectiva, No. 2 Facultad de Humanidades. Universidad del Valle. Octubre 1995
} 
y privados); las necesidades sociales que subyacen a dichas demandas, así como las respuestas que a ellas se ofrecen; los modos de vida de los usuarios; el análisis de las políticas sociales y su relación con el Trabajo Social; y las condiciones del trabajador-a social como trabajador y, finalmente, las consecuencias o los resultados mediatos de la acción profesional.

Lo anterior, contribuiría a profundizar en el objeto de intervención profesional y a develar y discutir los marcos teóricos-metodológicos subyacentes ${ }^{18}$.

3. Fortalecimiento de la investigación: la experiencia brasilera de los núcleos de investigación sobre líneas temáticas de interés para la profesión, podría ser una experiencia a implementar en las Escuelas de Trabajo Social. Los núcleos que son liderados por docentes, a los cuales se inscriben estudiantes (como ejercicio de iniciación científica), han contribuido a profundizar y a ampliar la producción de conocimiento en Trabajo Social (entre ellos el de las metodologías) y a cualificar a los-las estudiantes en investigación.

De esa forma, la investigación no sólo es un grupo de asignaturas, sino un proyecto de Escuela que orienta y atraviesa el proceso formativo y, a su vez, constituye una forma de articularse a procesos sociales concretos ${ }^{19}$.

Ello requiere, entre otras cosas, el aumento de la capacidad de respuesta de las Escuelas a las distintas convocatorias nacionales e internacionales de investigación y una revisión del componente de extensión universitaria.

${ }^{18}$ Entiéndase que el conocimiento no proviene de las prácticas profesionales, sino de la reflexión rigurosa sobre ella.

19 Algunos núcleos de investigación están articulados a las dinámicas locales. Resalto el de "Formaçao de lideranças em saúde", en la Escuela de Servicio Social de la Universidad Federal de Pernambuco, el cual constituye casi un observatorio del desarrollo de la participación social en salud. 


\section{BIBLIOGRAFÍA}

Ahumada, Consuelo. El Modelo Neoliberal y su Impacto en la Sociedad Colombiana, Bogotá: el Ancora Editores, 1996.

ARLWIN, NIDIA. JIMÉNEZ, MÓNICA., QUEZADA, MARGARITA. Un enfoque operativo de la metodología de Trabajo Social. Lumen Humanitas, Argentina, 6a. Edición, 1999

ANTUNES, RICARDo. Adeus ao Trabalho? Ensaio sobre as Metamorfoses e a Centralidade do Mundo do Trabalho, Campinas/SP: Cortez, ed. Unicamp, 1995.

Consejo Nacional para la Educación en Trabajo Social. Revista Colombiana de Trabajo Social. Manizales. No. 15, marzo 2001

Consejo Nacional para la Educación en Trabajo Social. Revista Colombiana de Trabajo Social. Manizales. No. 17, marzo 2003

Consejo Nacional para la Educación en Trabajo Social Revista Colombiana de Trabajo Social. Manizales. No.14, Bogotá 2000

Harvey, David. A Condição Pós-Moderna, São Paulo:Ed. Loyola, 1994

Runes, DAgoberto. Diccionario de filosofía. Ed. Grijalbo. 15ª edición, 1981

sarmiento, Libardo anzola. Utopía y Sociedad: Una Propuesta para el Próximo Milenio, Bogotá: Ed. Fescol, 1996.

STIGLiTZ, JOSEPH E. El malestar en la globalización. Ed. Taurus. Santa fe de Bogotá, 2002.

stiglitz, joseph e. morin, edgar. Castells, manuel. ext al. Pánico en la globalización. Fica. Colección el pez en la red, Bogotá, 2002.

HARDT, MICHAEL. NEGRI, ANTONIO. Empire. Library of Congress. United Status of America, 2000 HORACIO DA COSTA, MARÍA DALVA. "O elo invisível dos processo de trabalho no sistema único de saúde em Natal (RN). Um estudo sobre as particularidades do trabalho dos assistentes sociais na área da saúde pública nos nos 90”. Dissertação de Mestrado. Universidade Federal de Pernambuco, Brasil. 1998

SCHNEIDER A.W. HYER, K. and LUPTAK M. "Suggestions to Social Workers for Surviving in Managed Care”. Health \& Social Work. Volume 25, Number 4. November 2000.

GLOBERMAN, J. Hospital restructuring: positioning social work to manage changes. Social Work Health Care 1999; 28(4):13-30. 